\title{
OPEN Decreased choroidal and scleral thicknesses in highly myopic eyes with posterior staphyloma
}

\author{
Un Chul Park ${ }^{1,2 \bowtie}$, Eun Kyoung Lee ${ }^{1,2}$, Bo Hee Kim ${ }^{1,2}$ \& Baek-Lok Oh ${ }^{1,2}$
}

In this cross-sectional study, we investigated choroidal thickness (CT) and scleral thickness (ST) in highly myopic eyes and their associations with ocular factors. Patients underwent widefield sweptsource optical coherence tomography (OCT) to measure the CT and ST at the subfovea and $3000 \mu \mathrm{m}$ superior, inferior, temporal, and nasal to the fovea and macular curvature. A total of 237 eyes (154 patients) were included. At all five measurement points, thinner CTs and STs were associated with longer axial lengths $(r=-0.548$ to -0.357 , all $P<0.001)$ and greater macular curvatures $(r=-0.542$ to -0.305 , all $P<0.001$ ). The CT and ST were significantly thinner in eyes with posterior staphyloma than in those without at all measurement points (all $P \leq 0.006$ ) but did not differ between eyes with the wide macular and narrow macular type of staphyloma. Eyes with myopic maculopathy of category $\geq 3$ according to the International Meta-Analysis for Pathologic Myopia classification had significantly thinner CTs and STs than those with category $\leq 2$ (all $P \leq 0.005$ ). In highly myopic eyes, a decrease in the CT and ST was more pronounced in eyes with more structural changes, such as longer axial length, steeper macular curvature, and the presence of posterior staphyloma.

Pathologic myopia is one of the leading causes of irreversible visual impairment worldwide, particularly in East Asian countries where myopia is highly prevalent ${ }^{1-3}$. Visual impairment is mainly caused by myopic maculopathy, which refers to a range of chorioretinal changes in the posterior pole. Myopic maculopathy has been shown to progress over time to a higher category of atrophic change, leading to significant visual loss ${ }^{4-6}$. Although several clinical and ocular factors have revealed to have a significant association with the progression of myopic maculopathy, little is known about the exact mechanism involved in the development and progression of myopic maculopathy.

Recent observational longitudinal studies have shown that a greater axial elongation is significantly associated with the progression of myopic maculopathy, which suggests that structural change in the eyeball may be a major contributing factor for myopic maculopath ${ }^{4,7}$. In addition, posterior staphyloma, which indicates the localized outpouching of the eyeball wall at the posterior pole with a radius of less than the surrounding curvature, is associated with a higher prevalence of macular complications such as chorioretinal atrophy, myopic choroidal neovascularization, and myopic traction maculopathy ${ }^{8,9}$.

In this context, structural evaluation of the posterior pole may be helpful in elucidating the pathogenesis of macular complications in pathologic myopia. Furthermore, the influence of posterior staphyloma on choroidal thickness (CT) or scleral thickness (ST) remains unknown. In this study, we evaluated the association of CT and ST with the structural changes related to high myopia, especially the presence of posterior staphyloma.

\section{Methods}

Study population. In this cross-sectional study, the medical records of patients with high myopia who were consecutively examined using swept-source optical coherence tomography (OCT) at the Retina Center of the Seoul National University Hospital between January 2018 and July 2020 were reviewed. High myopia was defined as an axial length of $\geq 26.5 \mathrm{~mm}$ or a myopic refractive error $\geq-6.0$ diopters. The exclusion criteria were as follows: (1) poor quality of the swept-source OCT image, that is, image not clear due to eye movement or media opacity and image that did not include subfoveal choroid and sclera within the scan depth or not centered on the fovea; (2) other retinal disorders, such as diabetic retinopathy, retinal vascular diseases, age-related macular degeneration, and central serous chorioretinopathy; (3) a previous history of vitreoretinal surgery, photodynamic therapy, and anti-vascular endothelial growth factor (VEGF) treatment; or (4) active myopic choroidal

\footnotetext{
${ }^{1}$ Department of Ophthalmology, Seoul National University College of Medicine, 103 Daehak-ro, Jongno-gu, Seoul 110-799, Korea. ${ }^{2}$ Retinal Degeneration Research Laboratory, Seoul National University Hospital Biomedical Research Institute, Seoul, Korea. ${ }^{\square}$ email: ucpark@snu.ac.kr
} 
neovascularization. The protocol and study design were approved by the Institutional Review Board of the Seoul National University Hospital (IRB no. 2003-231-1115), and this study was conducted in accordance with the tenets of the Declaration of Helsinki. Informed consent was obtained from each participant for the study protocol and publication of identifying images in an online open-access publication.

Clinical examination. All patients underwent a comprehensive ophthalmic examination including bestcorrected visual acuity (BCVA), slit lamp examination, fundus examination, refractive error (spherical equivalent), axial length measurement, ultra-widefield retinal imaging, and widefield swept-source OCT. The axial length measurement was performed with the ocular biometry (IOLMaster; Carl Zeiss Meditec, Jena, Germany), and the ultra-widefield retinal image was taken with an Optos 200Tx scanning laser ophthalmoscope (Optos PLC, Scotland, UK) centered on the macula. Based on ultra-widefield retinal images, posterior staphyloma was determined by the presence of abnormal pigmentary changes in the pseudo-color image or an abnormal reflex in the red or green separation image indicating the staphyloma border ${ }^{8,10}$. When the presence of posterior staphyloma was ambiguous, widefield swept-source OCT images were also assessed to detect the staphyloma border, which is characterized by a gradual thinning of the choroid and an inward protrusion of the sclera ${ }^{11}$. The type of posterior staphyloma was determined based on the classification by Ohno-Matsui ${ }^{8}$, which classified the posterior staphyloma into five types (wide macular, narrow macular, peripapillary, nasal, and inferior) and others, according to its location and extent. According to the Meta-analysis of Pathologic Myopia (META-PM) Study Group classification, myopic maculopathy was classified into four categories: category 1, tessellated fundus; category 2, diffuse chorioretinal atrophy; category 3, patchy chorioretinal atrophy; and category 4, macular atrophy ${ }^{12}$. Three additional features, including lacquer cracks, Fuchs spots, and myopic choroidal neovascularization, were defined as plus lesions to supplement these categories.

Swept-source OCT measurement. Widefield swept-source OCT examination was performed using the PLEX Elite 9000 (Carl Zeiss Meditec, Dublin, CA) with an HD spotlight B-scan with a 16-mm scan length and a 3-mm scan depth. Some patients were examined using a protocol with a 6-mm scan depth after the OCT machine upgrade. Our scanning protocol included a pattern of radial scans along six meridians centered on the fovea. A single image was made up of 1024 A-line scans and averaged 100 times to yield a high-quality image. The thickness of the sclera and choroid was measured manually using a caliper provided by the OCT machine at the subfoveal region and $3000 \mu \mathrm{m}$ nasal, temporal, superior, and inferior to the fovea center. Considering that the dimension in the OCT images are affected by long axial length, measurement points of each eye were determined based on a previously reported formula: $t=3.382 \times 0.01306 \times($ axial length -1.82$) \times s$, where $t$ and $s$ represent the actual dimensions and measurements in the OCT image, respectively ${ }^{13}$. The CT was defined as the perpendicular distance between the outer edge of the hyperreflective line of the retinal pigment epithelium (RPE) and the choroid-scleral junction. The OCT images were maximally magnified when the choroid was extremely thin, but CT was determined as $0 \mu \mathrm{m}$ at points where the choroid was not visible. The ST was defined as the perpendicular distance between the choroid-scleral junction and outer scleral border. The thickness of the episclera, which appeared as a relatively uniform structure with a slightly lower reflectivity than the sclera, was not included in the ST measurement ${ }^{14}$. When a definite linear hyporeflective structure running within the sclera along its curvature, which suggests short or long posterior ciliary artery ${ }^{15}$, was observed, the hyporeflective portion within the sclera was not included in the ST. In addition, the curvature index, which is a recently proposed parameter to assess the macular curvature in myopic eyes ${ }^{16}$, was measured using the ImageJ software (NIH, Bethesda, MD). As a ratio of RPE line length compared to the straight distance between two points $6 \mathrm{~mm}$ apart centered on the fovea, greater curvature index represented a steeper macular curvature with more protrusion (see Supplementary Figure S1 online). The curvature indices obtained using six radial B scan images were averaged for analysis. In the analysis of subfoveal ST and curvature index, eyes with dome-shaped macula were excluded, which was defined as an inward bulge of the macular RPE of $>50 \mu \mathrm{m}$ above a presumed line of the RPE line at the bottom of the macular curvature.

Statistical analysis. Two independent retinal specialists (BO and $\mathrm{BHK}$ ) performed the grading of myopic maculopathy and posterior staphyloma and the measurement of CT, ST, and curvature index in a masked manner. Any discrepancies were adjudicated by a senior retinal specialist (UCP), and the measurements from the two graders were averaged for statistical analysis. Interobserver reproducibility of the CT, ST, and curvature index was assessed using intraclass correlation coefficients. Considering the invisibility of the posterior scleral border in some eyes, clinical and ocular characteristics were compared between eyes with and without any immeasurable points of ST. Correlations of CT and ST with age, axial length, and curvature index were evaluated. The CT, ST, and curvature index were compared according to the categories of myopic maculopathy or the presence of a posterior staphyloma using one-way analysis of variance or $t$-test, respectively. Post hoc analysis was performed using the Bonferroni method. All statistical analyses were performed using SPSS software version 22.0 (SPSS Inc., Chicago, IL), and $P$ values of $<0.05$ were considered statistically significant.

\section{Results}

Of the 372 eyes of 189 highly myopic patients who underwent swept-source OCT examination during the study period, 135 eyes were excluded: 40 eyes due to the poor quality of OCT images, 11 due to the presence of other chorioretinal disorders, 41 due to a history of intravitreal anti-VEGF treatment or photodynamic therapy, and 43 due to a history of vitreoretinal surgery. In total, 237 eyes of 154 patients were included in the analysis. The mean age was $63.0 \pm 11.6$ years (range, 24-89), and the mean axial length was $30.4 \pm 2.3 \mathrm{~mm}$ (range, 25.1-37.1). Baseline demographic and ocular characteristics of the study eyes are listed in Table 1. According to the META-PM 


\begin{tabular}{|c|c|c|c|c|c|c|}
\hline Parameters & Total & $\begin{array}{l}\text { Category } 1 \text { (fundus } \\
\text { tessellation) }\end{array}$ & $\begin{array}{l}\text { Category } 2 \text { (diffuse } \\
\text { atrophy) }\end{array}$ & $\begin{array}{l}\text { Category } 3 \text { (patchy } \\
\text { atrophy) }\end{array}$ & $\begin{array}{l}\text { Category } 4 \text { (macular } \\
\text { atrophy) }\end{array}$ & $P$-value \\
\hline $\begin{array}{l}\text { Number of eyes (\%)/ } \\
\text { patients }\end{array}$ & $\begin{array}{l}237 \text { eyes }(100.0 \%) / 154 \\
\text { patients }\end{array}$ & 63 eyes $(26.6 \%)$ & 95 eyes $(40.1 \%)$ & 61 eyes $(25.7 \%)$ & 18 eyes $(7.6 \%)$ & - \\
\hline Mean age, years (range) & $63.0 \pm 11.6(24$ to 89$)$ & $59.4 \pm 14.3(24$ to 89$)$ & $61.9 \pm 10.6(38$ to 85$)$ & $64.8 \pm 9.9(38$ to 81$)$ & $69.5 \pm 7.7(34$ to 83$)$ & 0.003 \\
\hline $\begin{array}{l}\text { Gender, number of eyes } \\
\text { (\%) of women }\end{array}$ & $\begin{array}{l}198 \text { eyes }(83.5 \%) / 128 \\
\text { women }\end{array}$ & 47 eyes $(74.6 \%)$ & 80 eyes $(84.2 \%)$ & 54 eyes $(88.5 \%)$ & 17 eyes $(94.4 \%)$ & 0.092 \\
\hline $\begin{array}{l}\text { Mean axial length, } \mathrm{mm} \\
\text { (range) }\end{array}$ & $30.4 \pm 2.3(25.1$ to 37.1$)$ & $28.4 \pm 1.6(25.1$ to 31.4$)$ & $30.4 \pm 2.0(25.7$ to 34.9$)$ & $31.9 \pm 2.0(28.4$ to 37.1$)$ & $31.1 \pm 2.4(28.2$ to 35.3$)$ & $<0.001$ \\
\hline $\begin{array}{l}\text { Mean refractive error, } \\
\text { diopter (range) }\end{array}$ & $\begin{array}{l}-15.4 \pm 5.4(-5.5 \text { to } \\
-25.0)\end{array}$ & $\begin{array}{l}-11.1 \pm 5.5(-5.5 \text { to } \\
-25.0)\end{array}$ & $\begin{array}{l}-15.7 \pm 4.6(-7.1 \text { to } \\
-23.8)\end{array}$ & $\begin{array}{l}-16.2 \pm 5.2(-9.3 \text { to } \\
-23.5)\end{array}$ & $\begin{array}{l}-14.7 \pm 4.3(-8.5 \text { to } \\
-20.3)\end{array}$ & 0.003 \\
\hline $\begin{array}{l}\text { Mean baseline BCVA, } \\
\text { logMAR (range, Snellen } \\
\text { equivalent) }\end{array}$ & $\begin{array}{l}0.55 \pm 0.55(-0.18 \text { to } 2.50 \\
20 / 71)\end{array}$ & $\begin{array}{l}0.30 \pm 0.28(-0.18 \text { to } 1.00 \\
20 / 40)\end{array}$ & $\begin{array}{l}0.46 \pm 0.40(-0.08 \text { to } 2.00 \\
20 / 58)\end{array}$ & $\begin{array}{l}0.60 \pm 0.56(-0.08 \text { to } 2.50 \\
20 / 80)\end{array}$ & $\begin{array}{l}1.66 \pm 0.64 \text { ( } 0.30 \text { to } 2.50 \\
20 / 914)\end{array}$ & $<0.001$ \\
\hline $\begin{array}{l}\text { Myopic choroidal neovas- } \\
\text { cularization }\end{array}$ & $18(7.6 \%)$ & $1(1.6 \%)$ & $3(3.2 \%)$ & $3(4.9 \%)$ & $11(61.1 \%)$ & $<0.001$ \\
\hline Lacquer crack & $58(24.5 \%)$ & $13(20.6 \%)$ & $29(30.5 \%)$ & $16(26.2 \%)$ & $0(0.0 \%)$ & 0.038 \\
\hline Dome-shaped macula & $39(16.5 \%)$ & $0(0.0 \%)$ & $15(15.8 \%)$ & $21(34.4 \%)$ & $3(16.7 \%)$ & $<0.001$ \\
\hline $\begin{array}{l}\text { Presence of posterior } \\
\text { staphyloma }\end{array}$ & $169(71.3 \%)$ & $30(47.6 \%)$ & $71(74.7 \%)$ & $53(86.9 \%)$ & $15(83.3 \%)$ & $<0.001$ \\
\hline
\end{tabular}

Table 1. Baseline characteristics and demographics of patients. BCVA best-corrected visual acuity, $\log M A R$ logarithm of minimal angle of resolution. ${ }^{a}$ Phakic eyes without history of refractive surgery.

Study Group classification, 63 (26.6\%), 95 (40.1\%), 61 (25.7\%), and 18 (7.6\%) eyes were graded as having myopic maculopathy of category 1 (fundus tessellation), category 2 (diffuse chorioretinal atrophy), category 3 (patchy chorioretinal atrophy), and category 4 (macular atrophy), respectively. Patients with a higher category of myopic maculopathy tended to be older, have a longer axial length, a greater myopic refractive error, and worse visual acuity. A plus lesion was present in 74 eyes (31.2\%). On swept-source OCT images, a dome-shaped macula was observed in 39 eyes (16.5\%).

Of the 1185 thickness measurement points in the 237 eyes, the entire thickness of the choroid was visible at all points. However, the posterior border of the sclera could not be detected on swept-source OCT images in 162 (13.7\%) points in 73 eyes (30.8\%); thus, ST was not measurable. These points were most frequently observed in eyes with myopic maculopathy of category 1 (113 points, $69.8 \%$ ), followed by those with category 2 (40 points, $24.7 \%), 3$ (6 points, $3.7 \%$ ), and 4 (3 points, 1.9\%). Topographically, ST was not measurable at the subfoveal, superior, inferior, temporal, and nasal regions in 21 (8.9\%), 54 (22.8\%), 20 (8.4\%), 47 (19.8\%), and 20 (8.4\%) eyes, respectively. When compared to the 164 eyes whose ST was measurable at all points, 73 eyes with one or more immeasurable points had a significantly shorter axial length $(P<0.001)$, lower category of myopic maculopathy $(P<0.001)$, lower frequency of posterior staphyloma $(P=0.028)$, and lower curvature index $(P=0.004)$. When comparing measurable thicknesses, eyes with any point of immeasurable ST had a significantly greater CT and ST at all points except the nasal CT (Table 2).

The CT and ST at all measurement points showed significant correlations with axial length and curvature index (all $P<0.001$; Table 3); a thin choroid and sclera were associated with a longer axial length and greater curvature index. Age was significantly correlated with subfoveal, superior, and temporal CT, but not with ST at any point. Significant correlations between CT and ST were found at all five measurement points (all $P<0.01$ ). At five measurement points, interobserver intraclass correlation coefficients were $\geq 0.892$ for CTs and $\geq 0.901$ for measurable STs. Interobserver intraclass correlation coefficients for the curvature index were 0.920 (see Supplementary Table S1 online).

Based on ultra-widefield retinal images, posterior staphyloma was observed in 169 eyes (71.3\%). The wide macular type was most commonly observed in 102 eyes (60.4\%), followed by narrow macular type in 53 eyes $(31.4 \%)$, peripapillary type in 7 eyes $(4.1 \%)$, inferior type in 5 eyes $(3.0 \%)$, and nasal type in 2 eyes $(1.2 \%)$. Eyes with posterior staphyloma had a significantly longer axial length $(P<0.001)$, a lower CT $(P \leq 0.003)$ and ST $(P \leq 0.006)$ at all measurement points, and a greater curvature index $(P<0.001)$ compared to those without posterior staphyloma (Table 4). However, there were no significant differences in axial length, CT, ST, and curvature index between the eyes with wide macular and narrow macular type posterior staphyloma (See Supplementary Table S2 online). In eyes with wide macular type staphyloma, four eyes showed combined staphylomas of peripapillary and narrow macular type. At all five measurement points, the CTs and STs of these four eyes were below the average of wide macular type staphyloma.

Figure 1 shows the CT and ST at five measurement points according to the categories of myopic maculopathy. For all parameters, one-way analysis of variance showed significant differences among the four categories. Eyes with category 1 had the greatest CT and ST at all points, while those with higher categories had lower thicknesses. Post hoc analysis by the Bonferroni method showed that category 1 differed from the other categories in all thickness parameters, except for category 2 in the subfoveal ST and nasal ST and from category 3 in subfoveal ST. Among the other categories, some parameters were different between categories 2 and 3 or between categories 2 and 4, but not between categories 3 and 4 . In 63 eyes with myopic maculopathy of META-PM category 1 in this study, 50 eyes $(79.4 \%)$ satisfied the cut-off CT of either $62 \mu \mathrm{m}$ at the subfovea to diagnose macular diffuse 


\begin{tabular}{|c|c|c|c|}
\hline \multirow[b]{2}{*}{ Parameters } & \multicolumn{2}{|l|}{ Visibility of posterior scleral border } & \multirow[b]{2}{*}{$P$-value } \\
\hline & Visible at all points & Invisible at any points & \\
\hline Number of eyes (\%) & $164(69.2 \%)$ & $73(30.8 \%)$ & - \\
\hline Mean age, years (range) & $62.4 \pm 11.0(24-84)$ & $63.0 \pm 12.9(27-89)$ & 0.739 \\
\hline Mean axial length, mm (range) & $31.1 \pm 2.1(25.8-37.1)$ & $28.8 \pm 2.0(25.1-33.8)$ & $<0.001$ \\
\hline $\begin{array}{l}\text { Mean baseline BCVA, logMAR (range, Snellen equiva- } \\
\text { lent) }\end{array}$ & $0.62 \pm 0.58(-0.08$ to $2.50,20 / 83)$ & $0.38 \pm 0.44(-0.18$ to $2.50,20 / 48)$ & 0.003 \\
\hline \multicolumn{4}{|l|}{ Category of myopic maculopathy } \\
\hline Category 1 (fundus tessellation) & $22(13.4 \%)$ & $41(56.2 \%)$ & \multirow{4}{*}{$<0.001$} \\
\hline Category 2 (diffuse atrophy) & $71(43.3 \%)$ & $24(32.9 \%)$ & \\
\hline Category 3 (patchy atrophy) & $55(33.5 \%)$ & $6(8.2 \%)$ & \\
\hline Category 4 (macular atrophy) & $16(9.8 \%)$ & $2(2.7 \%)$ & \\
\hline \multicolumn{4}{|l|}{ Choroidal thickness, $\mu \mathrm{m}$ (range) } \\
\hline Subfoveal & $25.0 \pm 16.2(0-95)$ & $55.1 \pm 39.0(0-187)$ & $<0.001$ \\
\hline Superior & $47.1 \pm 32.1(0-178)$ & $90.9 \pm 52.7(0-245)$ & $<0.001$ \\
\hline Inferior & $37.8 \pm 28.2(0-153)$ & $71.3 \pm 39.2(0-190)$ & 0.008 \\
\hline Temporal & $41.9 \pm 34.3(0-165)$ & $82.5 \pm 52.0(0-257)$ & $<0.001$ \\
\hline Nasal & $23.9 \pm 19.1(0-137)$ & $40.3 \pm 24.5(0-149)$ & 0.083 \\
\hline \multicolumn{4}{|l|}{ Scleral thickness, $\mu \mathrm{m}$ (range) $^{\mathrm{a}}$} \\
\hline Subfoveal $^{\mathrm{b}}$ & $261.2 \pm 86.9(103-501)$ & $336.8 \pm 87.1(125-570)$ & $<0.001$ \\
\hline Superior & $237.6 \pm 73.5(90-517)$ & $361.1 \pm 76.8(210-522)$ & $<0.001$ \\
\hline Inferior & $227.0 \pm 61.5(93-447)$ & $290.9 \pm 77.1(118-469)$ & $<0.001$ \\
\hline Temporal & $229.4 \pm 71.8(70-448)$ & $297.1 \pm 89.1(153-469)$ & $<0.001$ \\
\hline Nasal & $257.2 \pm 78.2(69-488)$ & $291.8 \pm 64.8(120-492)$ & 0.003 \\
\hline Presence of posterior staphyloma & $124(75.6 \%)$ & $45(61.6 \%)$ & 0.028 \\
\hline Curvature index (range) ${ }^{\mathrm{b}}$ & $1.049 \pm 0.032(1.011-1.241)$ & $1.035 \pm 0.027(1.007-1.181)$ & 0.003 \\
\hline
\end{tabular}

Table 2. Comparison of clinical characteristics according to the visibility of posterior scleral border. BCVA best-corrected visual acuity, $\log M A R$ logarithm of minimal angle of resolution. ${ }^{\text {a }}$ Included only points where scleral thickness was measurable. ${ }^{b}$ Excluded eyes with dome-shaped macula.

\begin{tabular}{|l|l|l|l|l|l|}
\hline \multicolumn{2}{|l}{ Parameters } & Age & Axial length & Curvature Index $^{\mathrm{a}}$ & Scleral thickness $^{* *}$ \\
\hline \multirow{4}{*}{ Choroidal thickness } & Subfoveal & $-0.224^{\dagger}$ & $-0.429^{\ddagger}$ & $-0.351^{\ddagger}$ & $0.278^{\mathrm{a}, \dagger}$ \\
\cline { 2 - 7 } & Superior & $-0.260^{\ddagger}$ & $-0.479^{\ddagger}$ & $-0.362^{\ddagger}$ & $0.389^{\ddagger}$ \\
\cline { 2 - 7 } & Inferior & -0.108 & $-0.548^{\ddagger}$ & $-0.321^{\ddagger}$ & $0.433^{\ddagger}$ \\
\cline { 2 - 7 } & Temporal & $-0.227^{\ddagger}$ & $-0.541^{\ddagger}$ & $-0.378^{\ddagger}$ & $0.403^{\ddagger}$ \\
\cline { 2 - 6 } & Nasal & -0.035 & $-0.537^{\ddagger}$ & $-0.305^{\ddagger}$ & $0.179^{\dagger}$ \\
\hline \multirow{5}{*}{ Scleral thickness } & Subfoveal & 0.053 & $-0.424^{\ddagger}$ & $-0.542^{\ddagger}$ & - \\
\cline { 2 - 7 } & Superior & -0.051 & $-0.428^{\ddagger}$ & $-0.423^{\ddagger}$ & - \\
\cline { 2 - 6 } & Inferior & -0.034 & $-0.505^{\ddagger}$ & $-0.423^{\ddagger}$ & - \\
\cline { 2 - 6 } & Temporal & 0.042 & $-0.543^{\ddagger}$ & $-0.339^{\ddagger}$ & - \\
\cline { 2 - 6 } & Nasal & -0.016 & $-0.357^{\ddagger}$ & $-0.408^{\ddagger}$ & - \\
\hline Curvature index ${ }^{\mathrm{a}}$ & & 0.019 & $0.458^{\ddagger}$ & - & - \\
\hline
\end{tabular}

Table 3. Correlation coefficients for the associations of choroidal and scleral thicknesses with age, axial length, and curvature index. ${ }^{\dagger} P$-value of correlation coefficient $<0.01 .{ }^{\ddagger} P$-value of correlation coefficient $<0.001$. ${ }^{a}$ Excluded eyes with dome-shaped macula.

choroidal atrophy or $56.5 \mu \mathrm{m}$ nasal to the fovea to diagnose peripapillary diffuse choroidal atrophy, as suggested in a recent OCT-based study ${ }^{17}$. In consideration of this finding, eyes with myopic maculopathy of category $\leq 2$ and $\geq 3$ were compared, and those with category $\leq 2$ had significantly greater CTs and STs at all measurement points (all $P \leq 0.005)$ and lower curvature index $(P=0.003)$. Representative OCT images of each category of myopic maculopathy are presented in the Figs. 2 and 3. 


\begin{tabular}{|c|c|c|c|}
\hline \multirow[b]{2}{*}{ Parameters } & \multicolumn{2}{|l|}{ Posterior staphyloma } & \multirow[b]{2}{*}{$P$-value } \\
\hline & Absent & Present & \\
\hline Number of eyes (\%) & $68(28.7 \%)$ & $169(71.3 \%)$ & - \\
\hline Mean age, years (range) & $62.0 \pm 13.9(27-89)$ & $62.8 \pm 10.6(24-85)$ & 0.616 \\
\hline Gender, number of eyes (\%) of women & $55(80.9 \%)$ & $143(84.6 \%)$ & 0.483 \\
\hline Mean axial length, mm (range) & $29.3 \pm 1.5(25.9-32.4)$ & $30.8 \pm 2.4(25.1-37.1)$ & $<0.001$ \\
\hline $\begin{array}{l}\text { Mean baseline BCVA, logMAR (range, Snellen equiva- } \\
\text { lent) }\end{array}$ & $0.47 \pm 0.54(-0.18$ to $2.50,20 / 59)$ & $0.58 \pm 0.56(-0.18$ to $2.50,20 / 76)$ & 0.206 \\
\hline Dome-shaped macula & $7(10.3 \%)$ & $32(18.9 \%)$ & 0.105 \\
\hline \multicolumn{4}{|l|}{ Choroidal thickness, $\mu \mathrm{m}$ (range) } \\
\hline Subfoveal & $46.9 \pm 39.3(0-187)$ & $29.2 \pm 21.7(0-133)$ & $<0.001$ \\
\hline Superior & $92.4 \pm 51.7(0-245)$ & $47.8 \pm 34.0(0-172)$ & $<0.001$ \\
\hline Inferior & $68.3 \pm 37.2(0-190)$ & $40.0 \pm 31.5(0-163)$ & $<0.001$ \\
\hline Temporal & $82.2 \pm 47.5(0-257)$ & $43.2 \pm 38.8(0-215)$ & $<0.001$ \\
\hline Nasal & $38.7 \pm 22.7(0-149)$ & $25.2 \pm 21.5(0-141)$ & 0.003 \\
\hline \multicolumn{4}{|l|}{ Scleral thickness, $\mu \mathrm{m}$ (range) } \\
\hline Subfoveal $^{\mathrm{a}}$ & $316.2 \pm 76.5(151-570)$ & $268.7 \pm 95.9(103-542)$ & 0.002 \\
\hline Superior & $304.0 \pm 81.5(157-522)$ & $230.3 \pm 76.5(90-460)$ & $<0.001$ \\
\hline Inferior & $276.7 \pm 78.9(93-455)$ & $231.0 \pm 67.0(101-469)$ & $<0.001$ \\
\hline Temporal & $276.3 \pm 65.9(140-411)$ & $226.6 \pm 77.7(70-469)$ & $<0.001$ \\
\hline Nasal & $288.9 \pm 69.1(139-488)$ & $257.1 \pm 76.9(69-492)$ & 0.006 \\
\hline Curvature index (range) ${ }^{\mathrm{a}}$ & $1.030 \pm 0.015(1.007-1.072)$ & $1.051 \pm 0.033(1.008-1.241)$ & $<0.001$ \\
\hline
\end{tabular}

Table 4. Choroidal and scleral thicknesses according to the presence of posterior staphyloma. BCVA best-

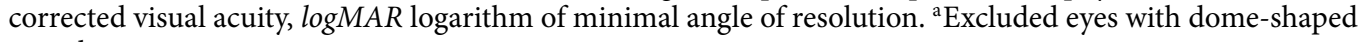
macula.
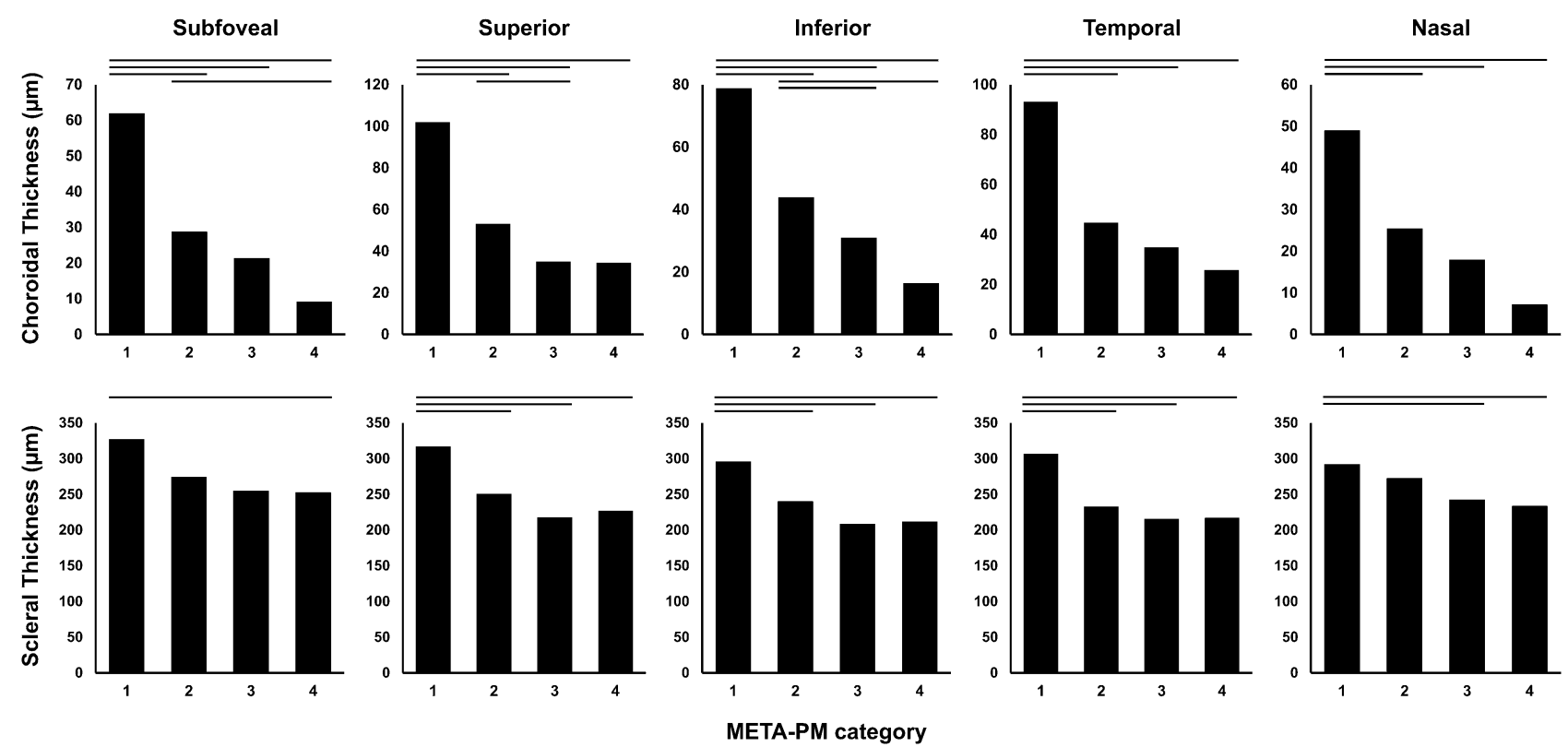

Figure 1. Choroidal and scleral thicknesses at the subfovea and at $3000 \mu \mathrm{m}$ superior, inferior, temporal, and nasal to the fovea according to the category of myopic maculopathy based on the Meta-analysis of Pathologic Myopia Study Group classification. Horizontal bars indicate statistically significant differences between the categories after correction for multiple comparisons.

\section{Discussion}

In this study, swept-source OCT imaging of highly myopic eyes revealed that decreased CT and ST were associated with a longer axial length and steeper macular curvature. In addition, CT and ST at all measurement points were significantly lower in eyes with posterior staphyloma than in those without. These findings suggest that structural alterations related to high myopia are associated with the decreased thickness of the choroid and sclera. 


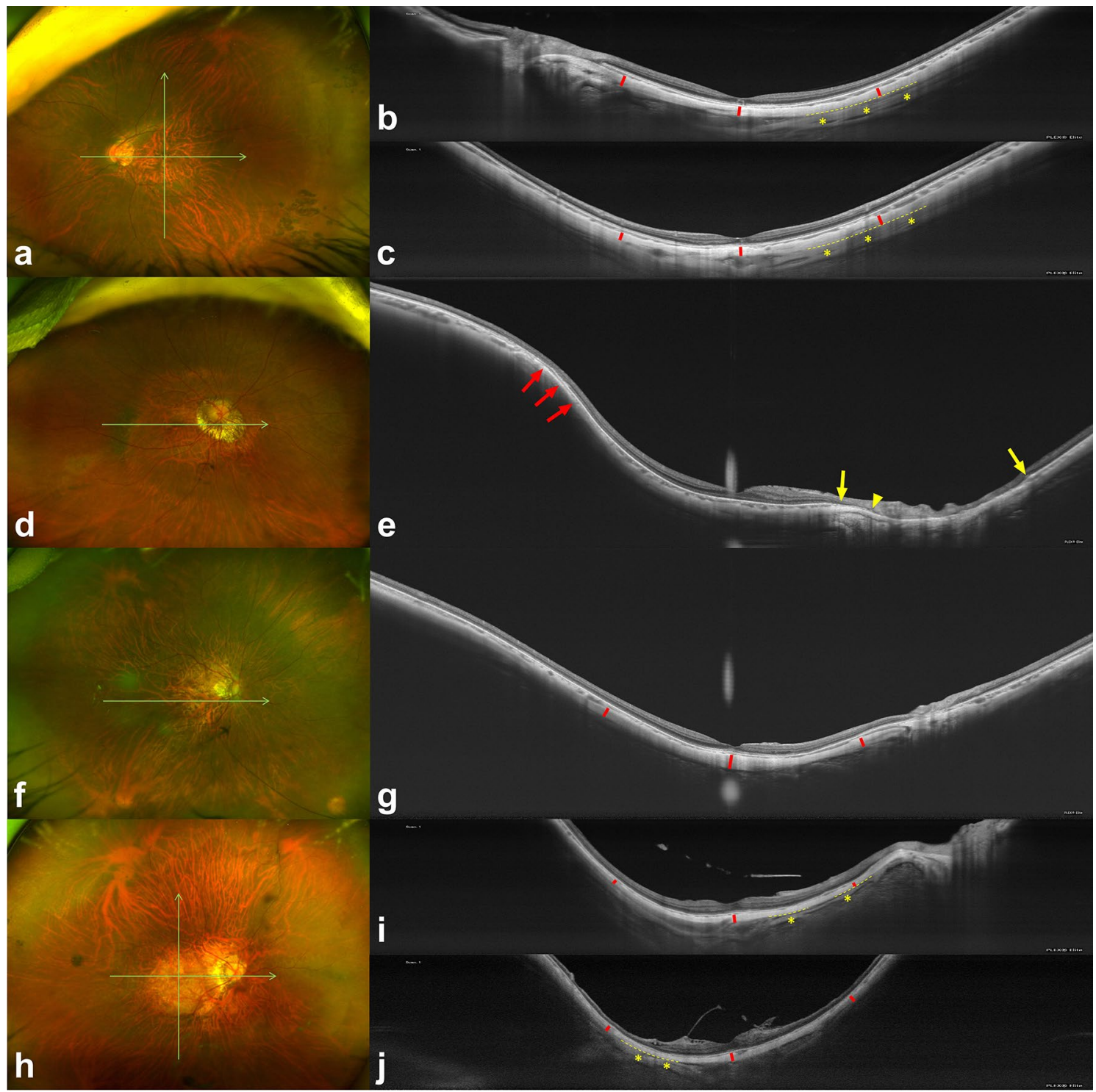

Figure 2. Images of highly myopic eyes with myopic maculopathy of category 1 (tessellated fundus) or 2 (diffuse chorioretinal atrophy). The green arrows represent the cross-section through which optical coherence tomography (OCT) scan was performed, and red bars represent scleral thicknesses at the fovea and at $3 \mathrm{~mm}$ from the fovea. In some images, the episcleral tissue appears as a uniform structure outside of the sclera with a slightly lower reflectivity (asterisks). Interfaces between the sclera and episcleral tissue are outlined with dashed lines. All OCT images were shown on a 1:1 $\mu \mathrm{m}$ scale. (a-c) A 24-year-old woman with tessellated fundus. The axial length was $30.63 \mathrm{~mm}$ and the best-corrected visual acuity (BCVA) was 20/32. The choroidal and scleral thicknesses at the foveal, superior, inferior, temporal, and nasal regions were 13, 122, 71, 81, $49 \mu \mathrm{m}$ and 214 , 234, 193, 205, $249 \mu \mathrm{m}$, respectively. (d,e) A 78-year-old woman with a mild peripapillary diffuse chorioretinal atrophy temporal to the disc. Axial length was $29.86 \mathrm{~mm}$ and BCVA was 20/25. Choroidal thickness at the foveal, temporal, and nasal regions was 55, 92, and $42 \mu \mathrm{m}$, respectively, but the posterior scleral border was not visible at any measurement point. An inward protrusion of the sclera and thinning of the choroid are observed at the edge of the staphyloma (red arrows). Note the margin of retinal pigment epithelium defects (yellow arrows) and end of the Bruch membrane (arrowhead). (f,g) A 63-year-old woman with peripapillary diffuse chorioretinal atrophy. Axial length was $30.07 \mathrm{~mm}$ and BCVA was 20/20. Choroidal and scleral thicknesses at the foveal, temporal, and nasal regions were 20,51, $17 \mu \mathrm{m}$ and 290, 211, $203 \mu \mathrm{m}$, respectively. (h-j) A 49-yearold woman with macular diffuse chorioretinal atrophy. Axial length was $34.55 \mathrm{~mm}$ and BCVA was 20/125. The choroidal and scleral thicknesses at the foveal, superior, inferior, temporal, and nasal regions were 19, 11, 12, 18, $14 \mu \mathrm{m}$ and 197, 168, 143, 115, $104 \mu \mathrm{m}$, respectively.

Swept-source OCT uses a longer wavelength light source, and improved tissue penetration allows for high-contrast imaging of the sclera and retrobulbar structures ${ }^{18}$. Compared to enhanced depth imaging by 


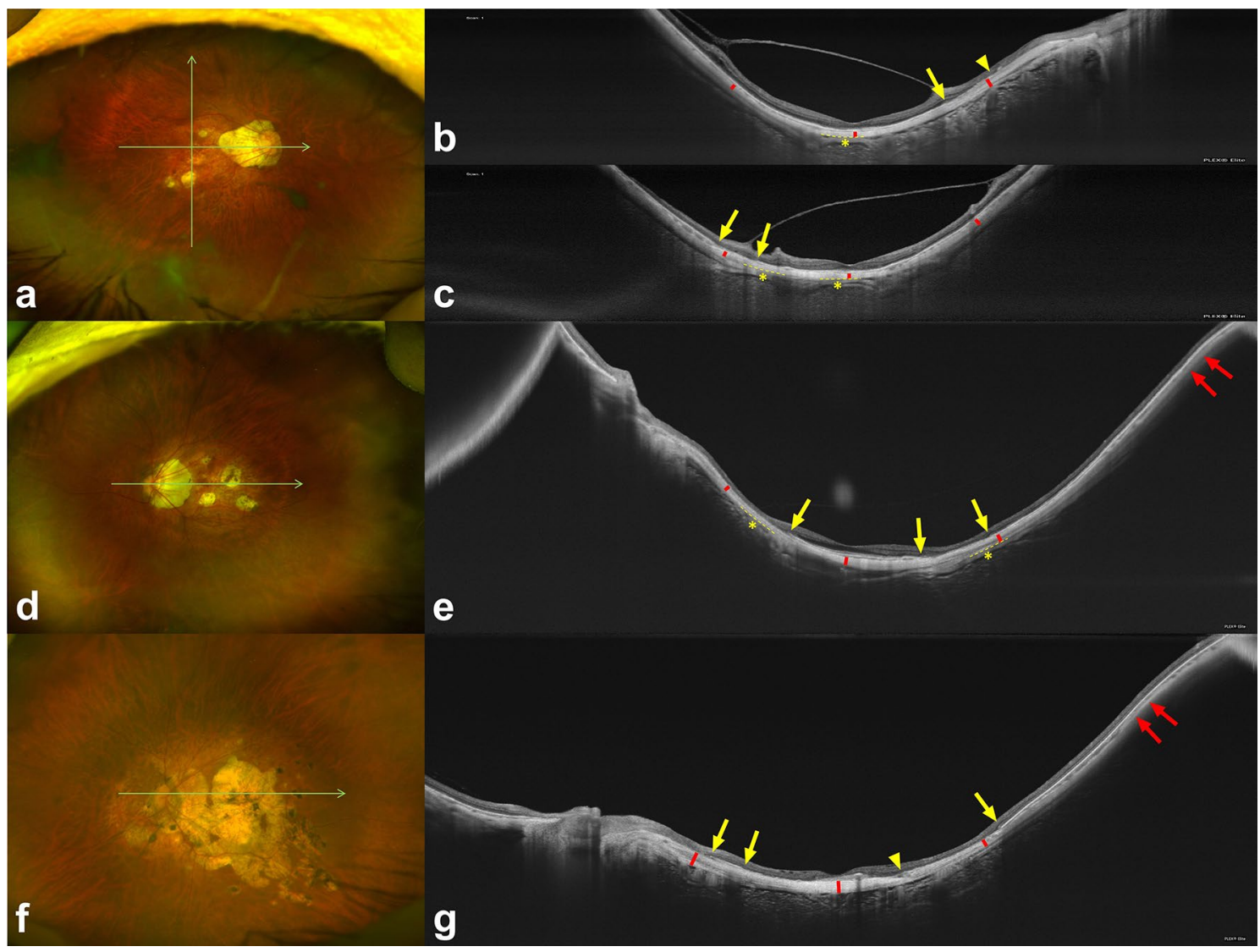

Figure 3. Images of highly myopic eyes with myopic maculopathy of category 3 (patchy chorioretinal atrophy) or 4 (macular atrophy). The green arrows represent the cross-section through which optical coherence tomography (OCT) scan was performed, and red bars represent scleral thicknesses at the fovea and $3 \mathrm{~mm}$ from the fovea. In some images, the episcleral tissue appears as a uniform structure outside of the sclera with a slightly lower reflectivity (asterisks). Interfaces between the sclera and episcleral tissue are outlined with dashed lines. All OCT images were shown on a 1:1 $\mu \mathrm{m}$ scale. (a-c) A 74-year-old man with patchy chorioretinal atrophy. The axial length was $33.92 \mathrm{~mm}$ and best-corrected visual acuity (BCVA) was 20/63. The choroidal and scleral thicknesses at the foveal, superior, inferior, temporal, and nasal regions were 15, 25, 34, 21, $0 \mu \mathrm{m}$ and 108, 126, $101,104,168 \mu \mathrm{m}$, respectively. Note the margin of retinal pigment epithelium defects (arrows) and end of Bruch membrane (arrowhead). (d,e) A 71-year-old woman with patchy chorioretinal atrophy. Axial length was $32.42 \mathrm{~mm}$ and BCVA was 20/40. Choroidal and scleral thicknesses at the foveal, temporal, and nasal regions were $30,15,0 \mu \mathrm{m}$ and $160,156,126 \mu \mathrm{m}$, respectively. Gradual thinning of the choroid is observed at the edge of the staphyloma (red arrows). Note the margin of retinal pigment epithelium defects (yellow arrows). (f,g) A 59-year-old man with choroidal neovascularization-related macular maculopathy. Axial length was $29.53 \mathrm{~mm}$ and visual acuity was count finger. On OCT, subretinal hyperreflective lesions at the fovea and extensive loss of the choroid and retinal pigment epithelium-Bruch membrane complex within an area of macular atrophy are observed. Note the margin of retinal pigment epithelium defects (yellow arrows) and crumpled remnants of the Bruch membrane (arrowhead). Gradual thinning of the choroid is observed at the edge of the staphyloma (red arrows). Choroidal and scleral thicknesses at the foveal, temporal, and nasal regions were $0,0,0 \mu \mathrm{m}$ and 217 , $145,226 \mu \mathrm{m}$, respectively.

spectral-domain OCT, swept-source OCT allows better visualization of the entire thickness of the sclera, which is thought to be limited by the thickness of the choroid ${ }^{19,20}$. In addition, it has a longer scan width and depth compared to conventional OCT. With the use of widefield swept-source OCT in this study, the presence of posterior staphyloma could be determined more reliably. More importantly, its longer scan depth enabled the measurements of CT and ST at the regions $3000 \mu \mathrm{m}$ from the fovea, even in eyes with a severely protruded posterior pole, which are usually out of the scan window and inverted within the image with conventional OCT. In this study, the entire thickness of the sclera was not visible in $13.7 \%$ of the measurement points, and $30.8 \%$ of patients had one or more points with immeasurable ST. This was comparable to the results of previous studies using swept-source OCT, which reported that the posterior scleral border was visible in $57-85 \%$ of highly myopic eyes but was less frequently or not visible in eyes with mild myopia or emmetropia ${ }^{19-21}$.

In a swept-source OCT study by Wong et al. $^{21}$, the choroid and sclera were thinner in eyes with myopic maculopathy of category $\geq 3$ than in eyes with category $\leq 2$ at multiple points in the posterior pole. However, the statistical significance was observed only in the subfoveal CT. In contrast, our results showed significantly greater CTs and STs at all measurement points, and this discrepancy may be due to the larger sample size in the 
present study. Based on the stronger correlation of CT with the severity of myopic maculopathy compared to ST, the authors considered vascular factors to be of more importance in the pathogenesis of myopic degeneration ${ }^{21}$. However, the results of the present study suggest that mechanical or structural changes reflected by scleral thinning also play an important role. The mechanism of choroidal and scleral thinning in axially elongated eyes has not been fully elucidated. The sclera has long been considered as the primary tissue of axial elongation based on the histologic and molecular findings of reduced diameter of collagen fibrils and decreased collagen synthesis in the sclera in animal models of myopia ${ }^{22-25}$. Recently, Jonas et al. suggested a plausible novel theory that axial elongation in highly myopic eyes may result from the production of Bruch membrane in the equatorial region and subsequent backward pushing of the Bruch membrane, which leads to the compression of the choroid and secondary thinning of the sclera ${ }^{26}$. Even in this hypothesis, the biomechanical property of the sclera seems to play an important role in the enlargement of the eyeball posterior to the equator, particularly the development of posterior staphyloma. A recent histological study on enucleated human eyes suggested that there is a possibility that local outpouching of the sclera may result from locally reduced scleral resistance caused by the altered arrangement of collagen fibril bundles ${ }^{27}$.

In this study, the thicknesses of the choroid and sclera at all measurement points were negatively correlated with the axial length and steepness of the posterior pole, which was represented by the curvature index. In addition, the present study shows that the choroid and sclera in eyes with posterior staphyloma were significantly thinner at all measurement points than in eyes without, confirming the histological findings that revealed decreased ST at the center of staphyloma in the enucleated highly myopic eyes ${ }^{27}$. To our knowledge, this is the first study that measured ST in vivo in highly myopic eyes with posterior staphyloma compared to those without, using swept-source OCT. These results suggest that highly myopic eyes with more protrusion at the posterior pole, particularly in the presence of localized outpouching of the posterior staphyloma, have a thinner sclera and choroid. Thinner choroid in eyes with posterior staphyloma may reflect that passive stretching can be also exerted on other inner ocular tissues within the staphyloma, such as the Bruch membrane, RPE, and retina. This is supported by previous findings that Bruch membrane defect or macular retinoschisis is mostly confined to the area within the staphyloma ${ }^{27,28}$.

Interestingly, when comparing the two most common types of posterior staphyloma, the wide macular and narrow macular type, they did not differ in the CT and ST at any measurement point or curvature index. Based on this, it may be assumed that the narrow macular type is not a less protruded form but an independent type of staphyloma. If the wide macular type is a more protruded form of staphyloma that progresses from the narrow macular type, one would expect further thinning of the choroid and sclera. Each highly myopic eye might have different a location and extent of the region with reduced scleral resistance resulting from altered collagen fibril arrangement ${ }^{29}$, and subsequent scleral outpouching may be confined only within this region, by which the type of posterior staphyloma is classified ${ }^{8}$. However, long-term changes in the type of staphyloma are still unknown, and it should be noted that patients with narrow macular type were significantly younger than those with wide macular type in this study. Although statistical analysis was not performed due to the small number of patients, eyes with combined staphylomas of peripapillary plus narrow macular type in this study had a thinner choroid and sclera compared to the simple wide macular type. This suggests that compound staphyloma might represent a more advanced form of staphyloma. Further longitudinal studies are required to verify the morphological changes in a posterior staphyloma.

This study has some limitations. First, because of the cross-sectional design of this study, it was difficult to determine the causal or temporal relationship between choroidal and scleral thinning in highly myopic eyes. Second, the presence of posterior staphyloma was mainly determined based on ultra-widefield retinal imaging, but this method has been reported to have high sensitivity and specificity for the detection of staphyloma ${ }^{8}$. Although widefield swept-source OCT images were also used to assess the presence of posterior staphyloma, the edge of the deep staphyloma may not be seen due to the limited scan depth. Third, thickness measurement of the extremely thin choroid may be less reliable due to the limited resolution of the OCT, although images were maximally magnified for CT measurement. Therefore, interobserver intraclass correlation coefficients for CT at measurement points with a thinner choroid, such as the subfoveal and nasal regions, were lower than those at other points, but were greater than 0.8 , indicating an excellent agreement. Intraobserver agreement was not evaluated, but interobserver agreements for all OCT measurements were excellent in this study.

In conclusion, this study demonstrated that CT and ST are more decreased in eyes with more structural changes of high myopia, especially in the presence of posterior staphyloma. Although the primary tissue of structural change in highly myopic eyes is not fully elucidated, a greater decrease in ST in eyes with posterior staphyloma suggests altered biomechanical properties of the sclera within the staphyloma. Further longitudinal studies will improve our understanding of the mechanism of myopic macular complications from the standpoint of structural change.

\section{Data availability}

The datasets generated during and/or analysed during the current study are available from the corresponding author on reasonable request.

Received: 14 December 2020; Accepted: 23 March 2021

Published online: 12 April 2021

\section{References}

1. Iwase, A. et al. Prevalence and causes of low vision and blindness in a Japanese adult population: the Tajimi Study. Ophthalmology 113, 1354-1362 (2006). 
2. Xu, L. et al. Causes of blindness and visual impairment in urban and rural areas in Beijing: the Beijing Eye Study. Ophthalmology 113(1134), e1-11 (2006).

3. Varma, R. et al. Prevalence and causes of visual impairment and blindness in Chinese american adults the Chinese american eye study. JAMA Ophthalmol. 134, 785-793 (2016).

4. Fang, Y. et al. Progression of myopic maculopathy during 18-year follow-up. Ophthalmology 125, 863-877 (2018).

5. Yan, Y. N. et al. Ten-year progression of myopic maculopathy: The Beijing eye study 2001-2011. Ophthalmology 125, 1253-1263 (2018).

6. Hayashi, K. et al. Long-term pattern of progression of myopic maculopathy: A natural history study. Ophthalmology 117, 1595-1611 (2010).

7. Li, Z. et al. Progression of myopic maculopathy in highly myopic chinese eyes. Investig. Ophthalmol. Vis. Sci. 60, 1096-1104 (2019).

8. Ohno-Matsui, K. Proposed classification of posterior staphylomas based on analyses of eye shape by three-dimensional magnetic resonance imaging and wide-field fundus imaging. Ophthalmology 121, 1798-1809 (2014).

9. Ruiz-Medrano, J. et al. Myopic maculopathy: Current status and proposal for a new classification and grading system (ATN). Prog. Retin. Eye Res. 69, 80-115 (2019).

10. Ohno-Matsui, K. et al. Features of posterior staphylomas analyzed in widefield fundus images in patients with unilateral and bilateral pathologic myopia. Retina 37, 477-486 (2017).

11. Shinohara, K. et al. Posterior staphylomas in pathologic myopia imaged by widefield optical coherence tomography. Investig. Ophthalmol. Vis. Sci. 58, 3750-3758 (2017).

12. Ohno-Matsui, K. et al. International photographic classification and grading system for myopic maculopathy. Am. J. Ophthalmol. 159, 877-883 (2015).

13. Nowroozizadeh, S. et al. Influence of correction of ocular magnification on spectral-domain OCT retinal nerve fiber layer measurement variability and performance. Investig. Ophthalmol. Vis. Sci. 55, 3439-3446 (2014).

14. Ohno-Matsui, K., Fang, Y., Morohoshi, K. \& Jonas, J. B. Optical coherence tomographic imaging of posterior episclera and tenon's capsule. Investig. Ophthalmol. Vis. Sci. 58, 3389-3394 (2017).

15. Ohno-Matsui, K., Akiba, M., Ishibashi, T. \& Moriyama, M. Observations of vascular structures within and posterior to sclera in eyes with pathologic myopia by swept-source optical coherence tomography. Investig. Ophthalmol. Vis. Sci. 53, 7290-7298 (2012).

16. Park, U. C., Ma, D. J., Ghim, W. H. \& Yu, H. G. Influence of the foveal curvature on myopic macular complications. Sci. Rep. 9, 16936 (2019).

17. Fang, Y. et al. OCT-based diagnostic criteria for different stages of myopic maculopathy. Ophthalmology 126, 1018-1032 (2019).

18. Ohno-Matsui, K. et al. Imaging retrobulbar subarachnoid space around optic nerve by swept-source optical coherence tomography in eyes with pathologic myopia. Investig. Ophthalmol. Vis. Sci. 52, 9644-9650 (2011).

19. Ohno-Matsui, K. et al. Association between shape of sclera and myopic retinochoroidal lesions in patients with pathologic myopia. Investig. Ophthalmol. Vis. Sci. 53, 6046-6061 (2012).

20. Park, H. Y. L., Shin, H. Y. \& Park, C. K. Imaging the posterior segment of the eye using swept-source optical coherence tomography in myopic glaucoma eyes: Comparison with enhanced-depth imaging. Am. J. Ophthalmol. 157, 550-557 (2014).

21. Wong, C. W., Phua, V., Lee, S. Y., Wong, T. Y. \& Cheung, C. M. G. Is choroidal or scleral thickness related to myopic macular degeneration?. Investig. Ophthalmol. Vis. Sci. 58, 907-913 (2017).

22. Li, H. et al. BMP-2 Is involved in scleral remodeling in myopia development. PLoS ONE 10, e0125219 (2015).

23. Tao, Y. et al. cAMP level modulates scleral collagen remodeling, a critical step in the development of myopia. PLoS ONE 8, e71441 (2013).

24. McBrien, N. A., Cornell, L. M. \& Gentle, A. Structural and ultrastructural changes to the sclera in a mammalian model of high myopia. Investig. Ophthalmol. Vis. Sci. 42, 2179-2187 (2001).

25. Gentle, A., Liu, Y., Martin, J. E., Conti, G. L. \& McBrien, N. A. Collagen gene expression and the altered accumulation of scleral collagen during the development of high myopia. J. Biol. Chem. 278, 16587-16594 (2003).

26. Jonas, J. B., Ohno-Matsui, K., Jiang, W. J. \& Panda-Jonas, S. Bruch membrane and the mechanism of myopization: A new theory. Retina 37, 1428-1440 (2017).

27. Jonas, J. B., Ohno-Matsui, K., Holbach, L. \& Panda-Jonas, S. Histology of myopic posterior scleral staphylomas. Acta Ophthalmol. 98, e856-e863 (2020).

28. Shinohara, K. et al. Ultrawide-Field OCT to Investigate Relationships between Myopic Macular Retinoschisis and Posterior Staphyloma. Ophthalmology 125, 1575-1586 (2018).

29. Wang, C., Xie, Y. \& Wang, G. The elastic modulus and collagen of sclera increase during the early growth process. J. Mech. Behav. Biomed. Mater. 77, 566-571 (2018).

\section{Author contributions}

Conceptualization of the work: U.C.P., Acquisition, analysis, or interpretation of data: B.H.K., B.O., Drafting of manuscript: U.C.P., E.K.L. Statistical analysis: U.C.P. Critical review of manuscript: B.O., E.K.L. All authors have approved the submitted version.

\section{Competing interests}

The authors declare no competing interests.

\section{Additional information}

Supplementary Information The online version contains supplementary material available at https:/doi.org/ 10.1038/s41598-021-87065-6.

Correspondence and requests for materials should be addressed to U.C.P.

Reprints and permissions information is available at www.nature.com/reprints.

Publisher's note Springer Nature remains neutral with regard to jurisdictional claims in published maps and institutional affiliations. 
(c) (i) Open Access This article is licensed under a Creative Commons Attribution 4.0 International cc) License, which permits use, sharing, adaptation, distribution and reproduction in any medium or format, as long as you give appropriate credit to the original author(s) and the source, provide a link to the Creative Commons licence, and indicate if changes were made. The images or other third party material in this article are included in the article's Creative Commons licence, unless indicated otherwise in a credit line to the material. If material is not included in the article's Creative Commons licence and your intended use is not permitted by statutory regulation or exceeds the permitted use, you will need to obtain permission directly from the copyright holder. To view a copy of this licence, visit http://creativecommons.org/licenses/by/4.0/.

(C) The Author(s) 2021 